\title{
Improve dosimetric outcome in stage III non-small-cell lung cancer treatment using spot-scanning proton arc (SPArc) therapy
}

\author{
Xiaoqiang Li', Peyman Kabolizadeh, Di Yan, An Qin, Jun Zhou, Ye Hong, Thomas Guerrero, Inga Grills, \\ Craig Stevens and Xuanfeng Ding ${ }^{*}$
}

\begin{abstract}
Background: To evaluate spot-scanning proton arc therapy (SPArc) and multi-field robust optimized intensity modulated proton therapy (RO-IMPT) in treating stage III non-small-cell lung cancer (NSCLC) patients.

Methods: Two groups of stage IIIA or IIIB NSCLC patients (group 1: eight patients with tumor motion less than 5 mm; group 2: six patients with tumor motion equal to or more than $5 \mathrm{~mm}$ ) were re-planned with SPArc and RO-IMPT. Both plans were generated using robust optimization to achieve an optimal coverage with $99 \%$ of internal target volume (ITV) receiving 66 Gy (RBE) in 33 fractions. The dosimetric results and plan robustness were compared for both groups. The interplay effect was evaluated based on the ITV coverage by single-fraction 4D dynamic dose. Total delivery time was simulated based on a full gantry rotation with energy-layer-switching-time (ELST) from 0.2 to $4 \mathrm{~s}$. Statistical analysis was also evaluated via Wilcoxon signed rank test.

Results: Both SPArc and RO-IMPT plans achieved similar robust target volume coverage for all patients, while SPArc significantly reduced the doses to critical structures as well as the interplay effect. Specifically, compared to RO-IMPT, SPArc reduced the average integral dose by $7.4 \%(p=0.001), V_{20}$, and mean lung dose by an average of $3.2 \%$ ( $\left.p=0.001\right)$ and 1.6 Gy (RBE) $(p=0.001)$, the max dose to cord by $4.6 \mathrm{~Gy}$ (RBE) $(p=0.04)$, and the mean dose to heart and esophagus by $0.7 \mathrm{~Gy}$ (RBE) ( $p=0.01)$ and $1.7 \mathrm{~Gy}(\mathrm{RBE})(p=0.003)$ respectively. The average total estimated delivery time was $160.1 \mathrm{~s}$, $213.8 \mathrm{~s}, 303.4 \mathrm{~s}, 840.8 \mathrm{~s}$ based on ELST of $0.2 \mathrm{~s}, 0.5 \mathrm{~s}, 1 \mathrm{~s}$, and $4 \mathrm{~s}$ for SPArc plans, compared with the respective values of $182.0 \mathrm{~s}(p=0.001), 207.9 \mathrm{~s}(p=0.22), 250.9 \mathrm{~s}(p=0.001), 509.4 \mathrm{~s}(p=0.001)$ for RO-IMPT plans. Hence, SPArc plans could be clinically feasible when using a shorter ELST.
\end{abstract}

Conclusions: This study has indicated that SPArc could further improve the dosimetric results in patients with locally advanced stage NSCLC and potentially be implemented into routine clinical practice.

Keywords: Spot scanning, Proton arc therapy, Lung cancer, Robust treatment planning

\section{Background}

Lung cancer remains the leading cause of cancer deaths for both men and women in the United States [1]. For patients with locally advanced lung cancer, radiotherapy alone or concurrent chemoradiotherapy has been widely used. Many trials have shown the benefits of concurrent chemoradiotherapy to improve the prognosis of patients, in terms of local control and survival [2-6]. However, the

\footnotetext{
* Correspondence: Xiaoqiang.Li@beaumont.org;

Xuanfeng.Ding@beaumont.org

Department of Radiation Oncology, Beaumont Health System, Royal Oak, MI, USA
}

(c) The Author(s). 2018 Open Access This article is distributed under the terms of the Creative Commons Attribution 4.0 International License (http://creativecommons.org/licenses/by/4.0/), which permits unrestricted use, distribution, and reproduction in any medium, provided you give appropriate credit to the original author(s) and the source, provide a link to the Creative Commons license, and indicate if changes were made. The Creative Commons Public Domain Dedication waiver (http://creativecommons.org/publicdomain/zero/1.0/) applies to the data made available in this article, unless otherwise stated.

associated toxicities to the surrounding normal tissues can be significant, which could limit the ability of target dose escalation to possibly improve the outcome [7-12].

Proton beam therapy, unlike photon radiotherapy, has inherent physical characteristics which are advantageous to treat disease sites such as locally advanced lung cancer, due to its sharp distal dose fall-off. Compared to passive scatter proton therapy (PSPT) [13], intensity modulated proton therapy (IMPT) has shown potentials to further reduce the dose to the adjacent normal tissues while maintaining similar or superior target coverage [14-17] in a more efficient way without having the need to use beam 
specific blocks or compensators. In IMPT, thousands of mono-energetic narrow beamlets ("spots") are optimized simultaneously and magnetically scanned to superpose a desired dose distribution. Although such active scanning delivery system provides the greatest flexibility to shape the target dose pattern, it can also be degraded by different uncertainties, including setup, range uncertainties [18-20], and the motion induced uncertainties [21-25].

Many studies have shown that robust optimization can effectively reduce the negative dosimetric impact from setup and range uncertainties compared to traditional margin approach in IMPT planning [26-32]. All studies on robust optimization, however, are still limited to few beam angles, due to low calculation speed and delivery efficiency. These obstacles limit our ability to further exploit the benefits of IMPT. With the recent development of spot-scanning arc therapy (SPArc) [33], which generates robust and delivery efficient spot scanning proton arc plans, we could potentially overcome the current dosimetric limitations $[34,35]$. Herein, we evaluate the potential benefits of SPArc for treating locally advanced non-small-cell lung cancer (NSCLC) relative to the conventional multi-field robust optimized IMPT (RO-IMPT) plans.

\section{Methods}

\section{Patient characteristics}

Fourteen patients with stage IIIA or IIIB NSCLC (Table 1) treated with intensity modulated radiotherapy (IMRT) at our institution previously were selected for this study. All patients received four-dimensional (4D) CT simulation using a helical CT scanner (Philips Brilliance Big Bore, Philips Healthcare System, Cleveland, OH). The internal

Table 1 Patient Characteristics

\begin{tabular}{|c|c|c|c|c|c|c|c|c|c|}
\hline \multirow[t]{2}{*}{ Group } & \multirow[t]{2}{*}{ ID } & \multirow[t]{2}{*}{ Age } & \multirow[t]{2}{*}{ Location } & \multirow[t]{2}{*}{ TNM stage } & \multirow[t]{2}{*}{ Stage } & \multirow[t]{2}{*}{ ITV(cc) } & \multicolumn{3}{|c|}{ Motion $(\mathrm{cm})$} \\
\hline & & & & & & & SI & $A P$ & LR \\
\hline \multirow[t]{8}{*}{1} & 1 & 56 & RUL & T3N2M0 & $\| \mathrm{A}$ & 164.1 & \multicolumn{3}{|c|}{$<0.5$} \\
\hline & 2 & 71 & LUL & T1bN2M0 & $\| \mathrm{A}$ & 107.0 & & & \\
\hline & 3 & 75 & LUL & T4N2M0 & $\| \mathrm{IIB}$ & 822.8 & & & \\
\hline & 4 & 49 & RUL & T2aN2M0 & $\| \mathrm{A}$ & 114.7 & & & \\
\hline & 5 & 64 & LLL & T3N2M0 & $\| \mathrm{A}$ & 345.3 & & & \\
\hline & 6 & 71 & LLL & T2bN3M0 & $\||| B$ & 265.4 & & & \\
\hline & 7 & 51 & LUL & T3N3M0 & $\| I B$ & 366.5 & & & \\
\hline & 8 & 56 & RLL & T4NOMO & $\| I A$ & 276.7 & & & \\
\hline \multirow[t]{6}{*}{2} & 9 & 66 & $R L L+R M L$ & T4N2M0 & $\| I \mid B$ & 350.6 & 0.5 & 0 & 0 \\
\hline & 10 & 76 & RML & T3N1M0 & $\| \mathrm{A}$ & 157.1 & 0.7 & 0.1 & 0.1 \\
\hline & 11 & 55 & LLL & T1aN2M0 & $\| \mathrm{A}$ & 734.6 & 0.6 & 0.1 & 0 \\
\hline & 12 & 75 & RUL & T2aN2M0 & $\| I A$ & 77.4 & 0.5 & 0.2 & 0.1 \\
\hline & 13 & 60 & LLL & T2N2M0 & $\| \mathrm{A}$ & 269.1 & 1.3 & 0.1 & 0.1 \\
\hline & 14 & 50 & $\mathrm{RLL}+\mathrm{RML}$ & T4N2M0 & IIIB & 401.8 & 1.2 & 0.1 & 0.3 \\
\hline
\end{tabular}

Abbreviations: RUL right upper lobe, LUL left upper lobe, LLL left lower lobe, $R L L$ right lower lobe, $R M L$ right middle lobe target volume (ITV) was defined on the average CT scan, which enveloped the gross target volumes (GTVs) on all individual respiratory phase CT scans. The ITV was then extracted with a $5 \mathrm{~mm}$ uniform expansion for clinical target volume (CTV). These fourteen patients were divided into two groups based on the extent of tumor motion. Group 1 contained eight patients with tumor motion less than $5 \mathrm{~mm}$, while group 2 included six patients with tumor motion equal to or more than $5 \mathrm{~mm}$. All the RO-IMPT plans were planned with three beams. The SPArc plans were generated using the SPArc algorithm with a partial arc starting from 10 degree initially with 2.5 degree sampling rate for the final plan. Both plans were optimized on average CT scan with the same following parameters: $3 \mathrm{~mm}$ uniform dose grid, 0.01 minimum monitor unit (MU) of spots, and $\pm 3.5 \%$ range, $5 \mathrm{~mm}$ setup uncertainties (21 scenarios), to minimize the dose to adjacent critical normal structures with an adequate coverage of 99\% ITV receiving 66 Gy (RBE) in 33 fractions. Similar objective constraints for organs at risks (OARs) were used for both plans.

\section{SPArc algorithm}

The SPArc algorithm was integrated into the worst-case scenario robust optimization in RayStation (version 5.0, RaySearch Laboratories AB, Stockholm, Sweden) to iteratively generate a proton arc plan with increasing control point sampling rate by the following steps: (1) control point re-sampling, (2) control point energy layers re-distribution, and (3) control point energy layers filtration. After the arc plan reaches a predefined control point sampling rate (e.g., 2-6 degrees), the SPArc algorithm continues (4) energy layers re-sampling and (5) spot number reduction to further assure the optimum solution while achieving the best treatment delivery efficiency. The details of such algorithm have been discussed by Ding et al. [33] .

\section{Plan evaluation}

For plan quality evaluation, the dose volume histograms (DVHs) of both target volume and OARs were generated on nominal dose distributions. The following dosimetric indices were compared: the dose at which $99 \%\left(D_{99 \%}\right)$ of the ITV volume was covered, the target maximum point dose $\left(D_{1 \%}\right)$, the percentages of the normalized volume of the total normal lung receiving more than $5 \mathrm{~Gy}, 10 \mathrm{~Gy}$, $20 \mathrm{~Gy}, 30 \mathrm{~Gy}\left(\mathrm{~V}_{5}, \mathrm{~V}_{10}, \mathrm{~V}_{20}, \mathrm{~V}_{30}\right)$, the normal lung mean dose, the spinal cord maximum point dose $\left(\mathrm{D}_{0.03 \mathrm{cc}}\right)$, the percentage of the normalized heart volume receiving more than $40 \mathrm{~Gy}\left(\mathrm{~V}_{40}\right)$, the heart mean dose, and the esophagus mean dose. The integral dose (ID) with unit in joule was also calculated according to Murshed et al. [36] as the following: 


$$
I D=\sum_{i} D_{i} * V_{i} * \rho_{i}
$$

where $D_{i}, V_{i}$, and $\rho_{i}$ are the dose, voxel volume, and density values of the $i$ th voxel in the body. The integral body dose describes the total energy imparted to the body.

The ITV coverage was further evaluated using the conformality index (CI), which was defined as [37]:

$$
C I=\frac{T V D p}{T V} \times \frac{T V D p}{V D p},
$$

where $T V D p, T V$, and $V D p$ are the target volume covered by the prescribed dose, target volume, and the volume enclosed by the prescription isodose line respectively.

In terms of plan robustness quantification, both SPArc and RO-IMPT plans were re-calculated with $5 \mathrm{~mm}$ isocenter shift in the anterior-posterior, superior-inferior, and right-left directions under nominal proton beam range, with $+3.5 \%$ and $-3.5 \%$ proton beam ranges uncertainties, corresponding to total of 21 dose distribution scenarios. The DVHs for all the scenarios were plotted for comparison. The root-mean-square dose (RMSD) for each voxel was calculated, and the RMSD volume histograms (RVHs) and the areas under the RVH curve (AUC) [38] were computed for both target volume and OARs to compare the robustness. The smaller the AUC value was, the more robust the plan for the corresponding organ was.

\begin{tabular}{|c|c|c|c|c|}
\hline Structures & Value & SPArC & RO-IMPT & $p$ value \\
\hline \multirow[t]{2}{*}{ ITV } & $\mathrm{D}_{99 \%}(\mathrm{~Gy})$ & 66 & 66 & $\mathrm{~N} / \mathrm{A}$ \\
\hline & $D_{1 \%}(G y)$ & 70.9 & 71.1 & 0.22 \\
\hline \multirow[t]{5}{*}{ Total Lung } & $V_{5}(\%)$ & 20.4 & 25.0 & 0.001 \\
\hline & $V_{10}(\%)$ & 16.2 & 20.4 & 0.001 \\
\hline & $V_{20}(\%)$ & 12.9 & 16.1 & 0.001 \\
\hline & $V_{30}(\%)$ & 10.8 & 13.4 & 0.001 \\
\hline & Mean(Gy) & 7.9 & 9.5 & 0.001 \\
\hline Cord & $\mathrm{D}_{0.03 c c}(\mathrm{~Gy})$ & 17.8 & 22.4 & 0.04 \\
\hline \multirow[t]{2}{*}{ Heart } & $V_{40}(\%)$ & 4.4 & 5.0 & 0.03 \\
\hline & Mean(Gy) & 4.2 & 4.9 & 0.01 \\
\hline Esophagus & Mean(Gy) & 12.9 & 14.6 & 0.003 \\
\hline ID & $(J)$ & 72.0 & 77.3 & 0.001 \\
\hline $\mathrm{Cl}$ & & 0.70 & 0.68 & 0.33 \\
\hline DT (ELST $0.2 \mathrm{~s}$ ) & (s) & 160.1 & 182.0 & 0.001 \\
\hline DT (ELST 0.5 s) & (s) & 213.8 & 207.9 & 0.22 \\
\hline DT (ELST $1 \mathrm{~s})$ & (s) & 303.4 & 250.9 & 0.001 \\
\hline DT (ELST 2 s) & (s) & 482.5 & 337.1 & 0.001 \\
\hline DT (ELST 4 s) & (s) & 840.8 & 509.4 & 0.001 \\
\hline
\end{tabular}

Table 2 Average dosimetric results for the fourteen patients

Abbreviations: ITV internal target volume, $\mathrm{Cl}$ conformality index, ID integral dose, DT estimated delivery time, ELST energy layer switch time
For patients in group 2 with tumor motion equal to or more than $5 \mathrm{~mm}$, the interplay effect were evaluated based on the average ITV coverage via the single-fraction 4D dynamic dose calculation without considering rescanning for different starting respiratory phases [23]. This 4D dynamic dose was calculated by synchronizing the breathing cycle with the machine delivery pattern $[23,25,39]$ (e.g., assigning the spots on different respiratory phases of the CT simulation scan based on the time scale), and then accumulated via the deformable image registration on the expiration phase for evaluation, assuming the energy-layer-switchingtime (ELST) of $1 \mathrm{~s}$ and a regular respiratory breathing period of $4.5 \mathrm{~s}$.

The comparisons of treatment delivery efficiency between SPArc and RO-IMPT plans were simulated based on a full gantry rotation (360 degrees) with 1 rotation per minute rotation speed, $2 \mathrm{~ms}$ spot switching time, and ELST of $0.2 \mathrm{~s}, 0.5 \mathrm{~s}, 1 \mathrm{~s}, 2 \mathrm{~s}$, and $4 \mathrm{~s}$ [33]. All the results were statistically analyzed with non-parametric Wilcoxon signed rank test using SPSS 19.0 software

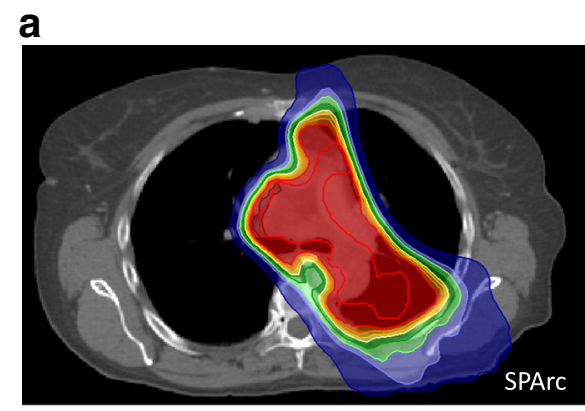

b
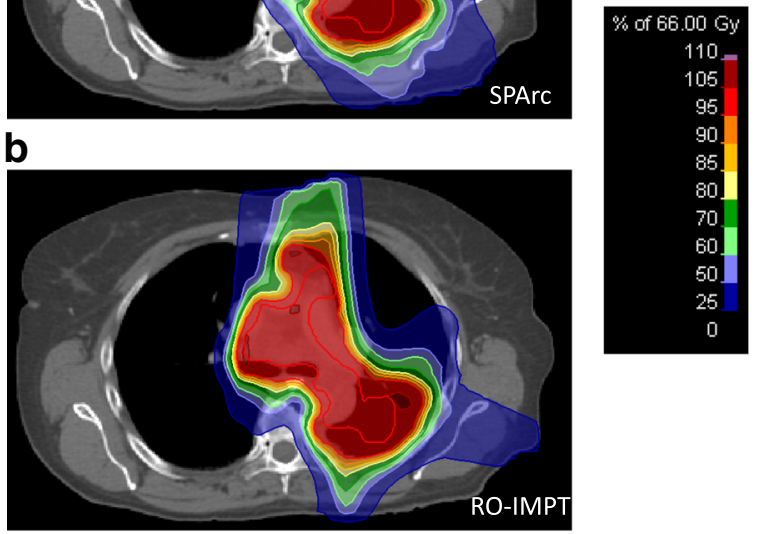

C

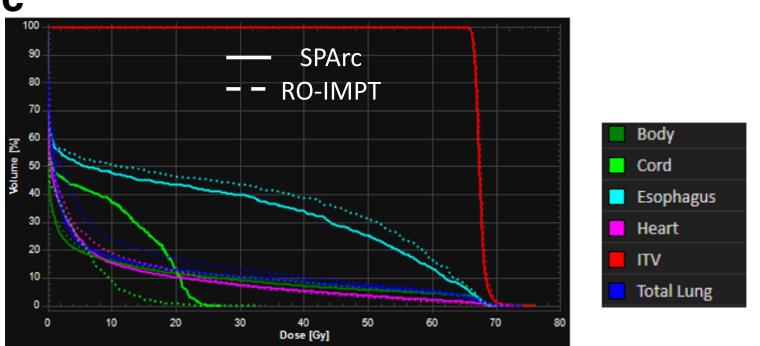

Fig. 1 Dose distributions of (a) SPArc and (b) RO-IMPT plans, and the corresponding DVHs (solid line for SPArc, dashed line for RO-IMPT) comparisons for patient 6 
(International Business Machines, Armonk, New York), and $p$ values equal to or less than 0.05 were considered statistically significant.

\section{Results}

\section{Dosimetric improvement}

Table 2 shows the average dosimetric parameters of fourteen patients comparing SPArc and RO-IMPT plans. With similar target coverage, in terms of $\mathrm{D}_{99 \%}, \mathrm{D}_{1 \%}$ dose to the target volume and conformality index, SPArc was able to significantly reduce the dose to all the critical structures evaluated. Specifically, SPArc plans decreased the average integral dose from $77.3 \mathrm{~J}$ to $72.0 \mathrm{~J}$, by $7.4 \%(p=0.001)$ compared to RO-IMPT plans. The average $\mathrm{V}_{5}, \mathrm{~V}_{10}, \mathrm{~V}_{20}$, $\mathrm{V}_{30}$ and mean lung dose for SPArc plans were 20.4\%, $16.2 \%, 12.9 \%, 10.8 \%$, and 7.9 Gy (RBE). While comparing to RO-IMPT plans, SPArc reduced the average $V_{5}$, $\mathrm{V}_{10}, \mathrm{~V}_{20}, \mathrm{~V}_{30}$, and mean lung dose by $4.6 \%(p=0.001)$, $4.2 \% \quad(p=0.001), 3.2 \% \quad(p=0.001), 2.6 \% \quad(p=0.001)$, and $1.7 \mathrm{~Gy}(\mathrm{RBE})(p=0.001)$ respectively. In terms of other OARs, SPArc reduced the max dose to cord by 4.6 Gy $(\mathrm{RBE})(p=0.04)$, the mean dose to heart and esophagus by 0.7 Gy (RBE) $(p=0.01)$ and 1.7 Gy (RBE) $(p=0.003)$ respectively compared to RO-IMPT plans. Figure 1 shows an example (patient 6) for both plans at nominal position.

\section{Robustness quantification}

Figure 2 shows the DVHs for target volume and OARs in all 21 scenarios for SPArc and RO-IMPT plans for patient 6 . Both plans could achieve an adequate coverage of at least $95 \%$ of ITV with the prescription dose under such uncertainties. Figure 3 shows the corresponding RVHs comparison for patient 6 (Fig. 3a) and the average mean AUC index of the fourteen cases and the corresponding $p$ values (Fig. 3b). The average AUC index of the ITV, were very similar, with the corresponding average AUC values of 0.87 and 0.84 Gy $(\mathrm{RBE})(p=0.27)$ respectively for both plans. SPArc could significantly reduce the
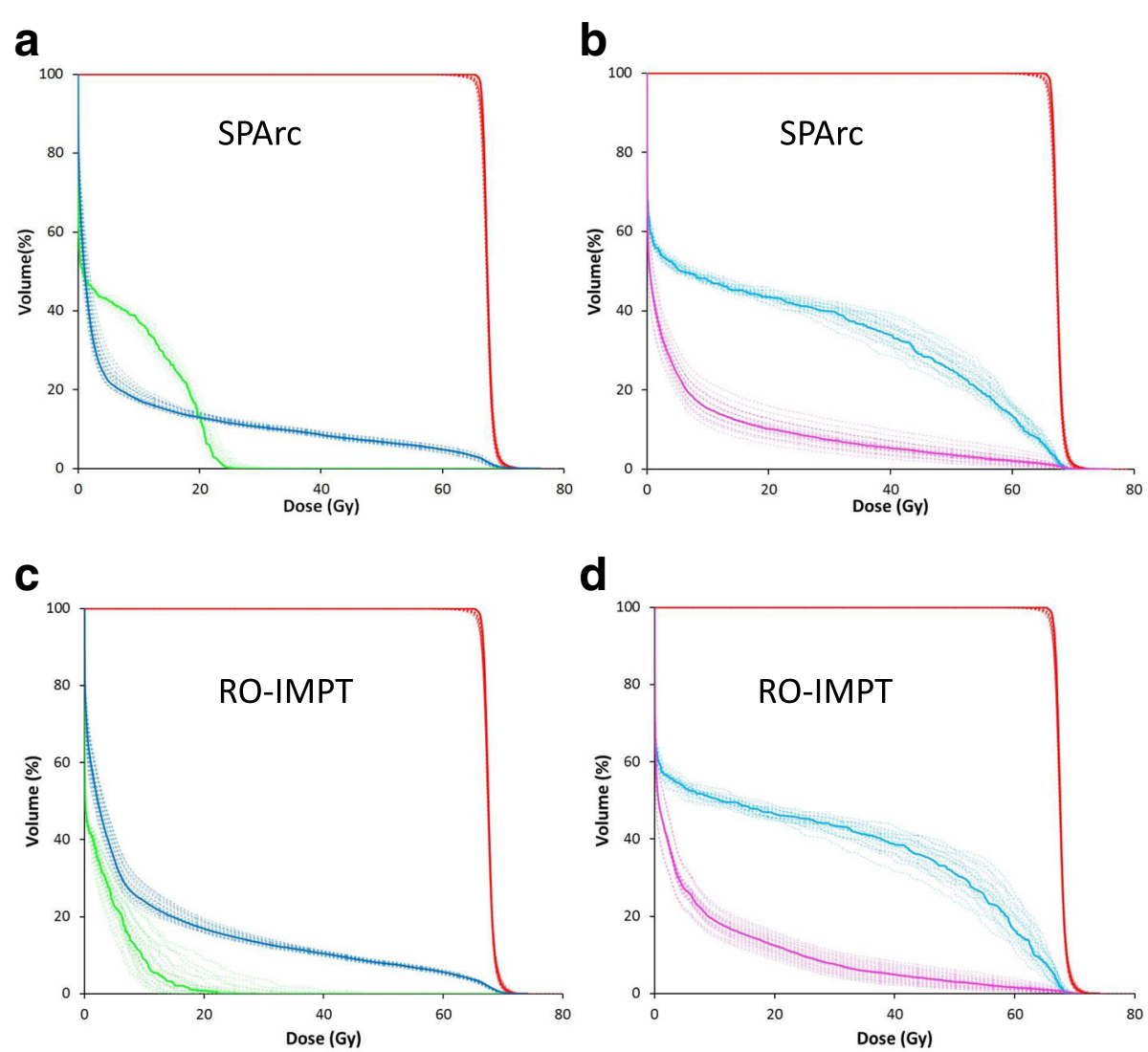

\section{-ITV —Cord -Esophagus - Heart —Total Lung}

Fig. 2 The dose distributions of (a, b) SPArc and (c, d) RO-IMPT plans for nominal position (solid line) and 20 scenarios of uncertainties (dashed line) for patient 6 

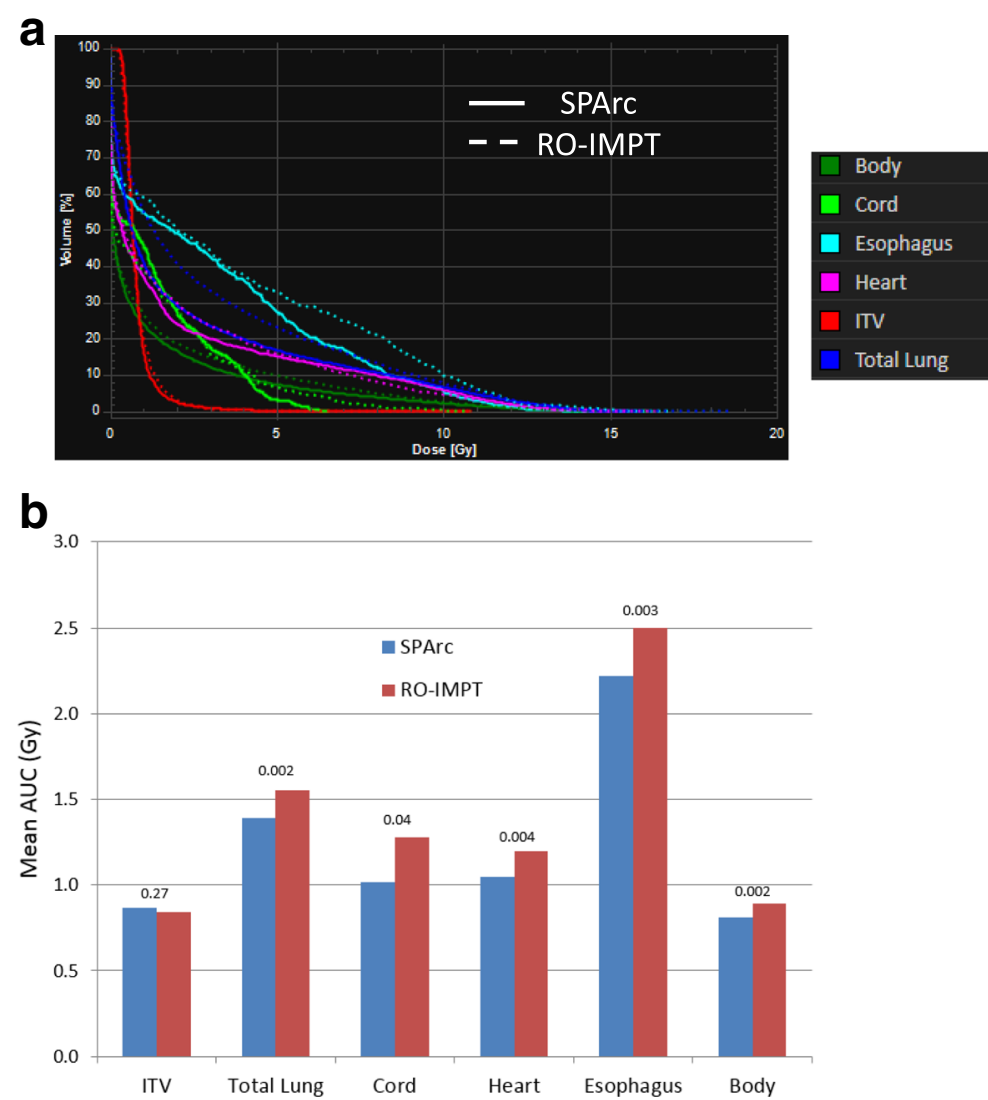

Fig. 3 a The root-mean-square dose (RMSD) volume histograms of SPArc (solid line) and RO-IMPT (dashed line) for patient 6. $\mathbf{b}$ The average areas under the RVH curve (AUC) for fourteen patients (b) with p-values on the top of the columns

average AUC values from 1.55 Gy $(\mathrm{RBE})$ to $1.39 \mathrm{~Gy}(\mathrm{RBE})$ $(p=0.002), 1.28 \mathrm{~Gy}(\mathrm{RBE})$ to $1.02 \mathrm{~Gy}(\mathrm{RBE})(p=0.04)$, $1.20 \mathrm{~Gy}(\mathrm{RBE})$ to $1.05 \mathrm{~Gy}(\mathrm{RBE})(p=0.004), 2.50 \mathrm{~Gy}(\mathrm{RBE})$ to $2.22 \mathrm{~Gy}(\mathrm{RBE})(p=0.003), 0.89 \mathrm{~Gy}(\mathrm{RBE})$ to $0.81 \mathrm{~Gy}$ (RBE) ( $p=0.002)$ for total normal lung, spinal cord, heart, esophagus, and integral body dose respectively.

\section{Delivery time}

Table 2 lists the estimated delivery time per fraction for both SPArc and RO-IMPT plans for various ELST. For the proton centers with ELST equaling to $4 \mathrm{~s}$, the estimated delivery time ratios between SPArc to RO-IMPT plans was 1.65 (840.8 s vs. $509.4 \mathrm{~s}$ ), with the corresponding time significantly being longer $(p=0.001)$ for SPArc plans. As the ELST gets shorter to $0.5 \mathrm{~s}$, the delivery time was still longer for SPArc plans but the differences were not statistically significant. With the ELST of $0.2 \mathrm{~s}$, the SPArc plan could be delivered in a shorter time when compared to RO$\operatorname{IMPT}(p=0.001)$.

\section{The effect of target movement and interplay}

SPArc has the potential to significantly reduce the interplay effect. More specifically, the average dose covering
95\% of ITV via single-fraction 4D dynamic dose without considering rescanning was 64.5 Gy for SPArc compared to the value of $62.0 \mathrm{~Gy}(p=0.01)$ for RO-IMPT. Figure $4 \mathrm{a}, \mathrm{b}$ shows an example of 4D dynamic dose calculation of SPArc versus RO-IMPT plans for patient 13. In this evaluation, it is clearly noted that SPArc reduced the cold spots and hot spots induced by motion in the target volume. Figure 4c showed the boxplot of the doses encompassing 95\% ( $\left.\mathrm{D}_{95 \%}\right)$ of ITV on exhalation phase for group 2 patients with different starting breathing phase. The median and range of the $D_{95 \%}$ are significantly improved via SPArc. Hence SPArc could effectively not only improve the target volume coverage but also decrease the extent of impact associated with respiratory phase treatment starting point on target coverage.

\section{The calculation speed of SPArc plan}

The average calculation time required to generate a SPArc plan is $4 \mathrm{~h}$, ranging from 3 to $6 \mathrm{~h}$ depending on the size of the target volume. This simulation was performed on a 64-bit workstation with an Intel Quad-Core processor (TM i5-4590 CPU @ 3.30 GHz) and 64 GB RAM. 
a

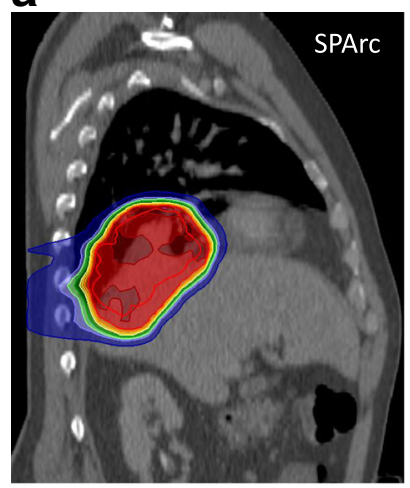

b

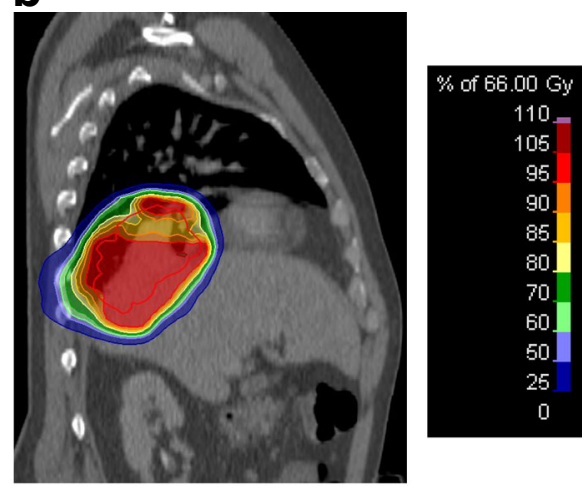

C

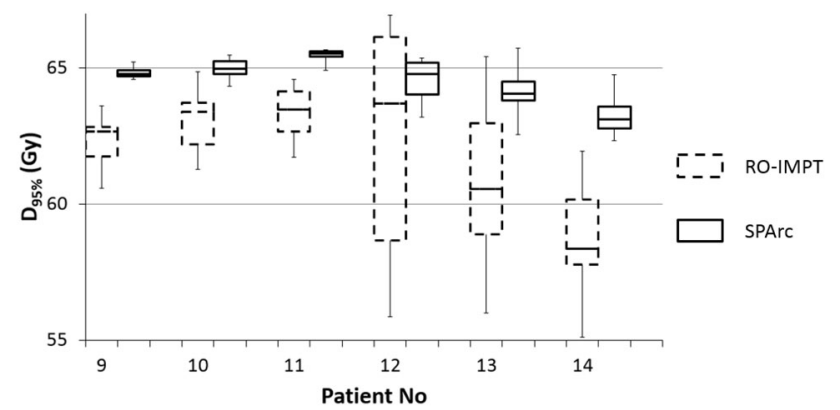

Fig. 4 Single-fraction 4D dynamic dose distributions without considering rescanning on exhale phase for (a) SPArc and (b) RO-IMPT plan for patient 13 and the boxplot of the doses encompassing 95\% (D95\%) ITV on exhalation phase for group 2 patients based on single-fraction 4D dynamic dose simulated with different starting breathing phase $(\mathbf{c})$

\section{Discussion}

This study evaluated the dosimetric benefits of using SPArc in treatment of stage III NSCLC patients. These results suggest that SPArc is able to significantly reduce the dose to all critical structures evaluated, while maintaining similar or superior target volume coverage and plan robustness compared to RO-IMPT plans. It is important to note that such additional organ dose sparing could potentially grant the clinician the ability to escalate the dose to the target volume safely. Although RTOG 0617 and a recent meta-analysis of randomized trials $[40,41]$ did not show a benefit of dose escalation in the chemoradiotherapy setting, the negative results could be partially related to high levels of toxicity, as dose escalation still appears to improve survival in the radiation alone setting. RTOG 0617 showed a strong association between overall survival and higher heart dose, especially the heart volume receiving more than 40 Gy [42]. Furthermore, the secondary analysis showed using IMRT planning also resulted in a significant reduction in grade 3 or higher pneumonitis. With techniques such as SPArc, shown here to further reduce lung and other normal tissues doses while concurrently reducing interplay effects related to respiratory tumor motion which may be critical in proton delivery, there may be further potential to dose escalate the target volume without increasing toxicity or mortality but potentially improving control or survival. As national cooperative groups compare proton to photon irradiation with survival endpoints, such as in the on-going RTOG 1308 and protons become more readily available in the community, these issues will become increasingly important.

One counterintuitive result of our study is that SPArc plans could significantly decrease the integral body dose compared to RO-IMPT plans despite that SPArc generally uses hundreds of beams entering from different angles. One explanation is that the iteratively energy layer selection algorithm of SPArc optimization tends to filter the highest energy of each beam angle and superimpose the dose contributions from other beam angles to cover the distal extent of the target volume to produce a most robust plan. Figure 5 a plots the MUs distributions versus energy of both SPArc and RO-IMPT plans for patient 6. As it is shown, the SPArc plan only used energies higher than $82.6 \mathrm{MeV}$ and lower than $164.5 \mathrm{MeV}$ while the RO-IMPT delivered a portion of the plan using energies higher than 164.5 MeV. In order to quantitatively illustrate this concept in more details, the calculated total energy 
a
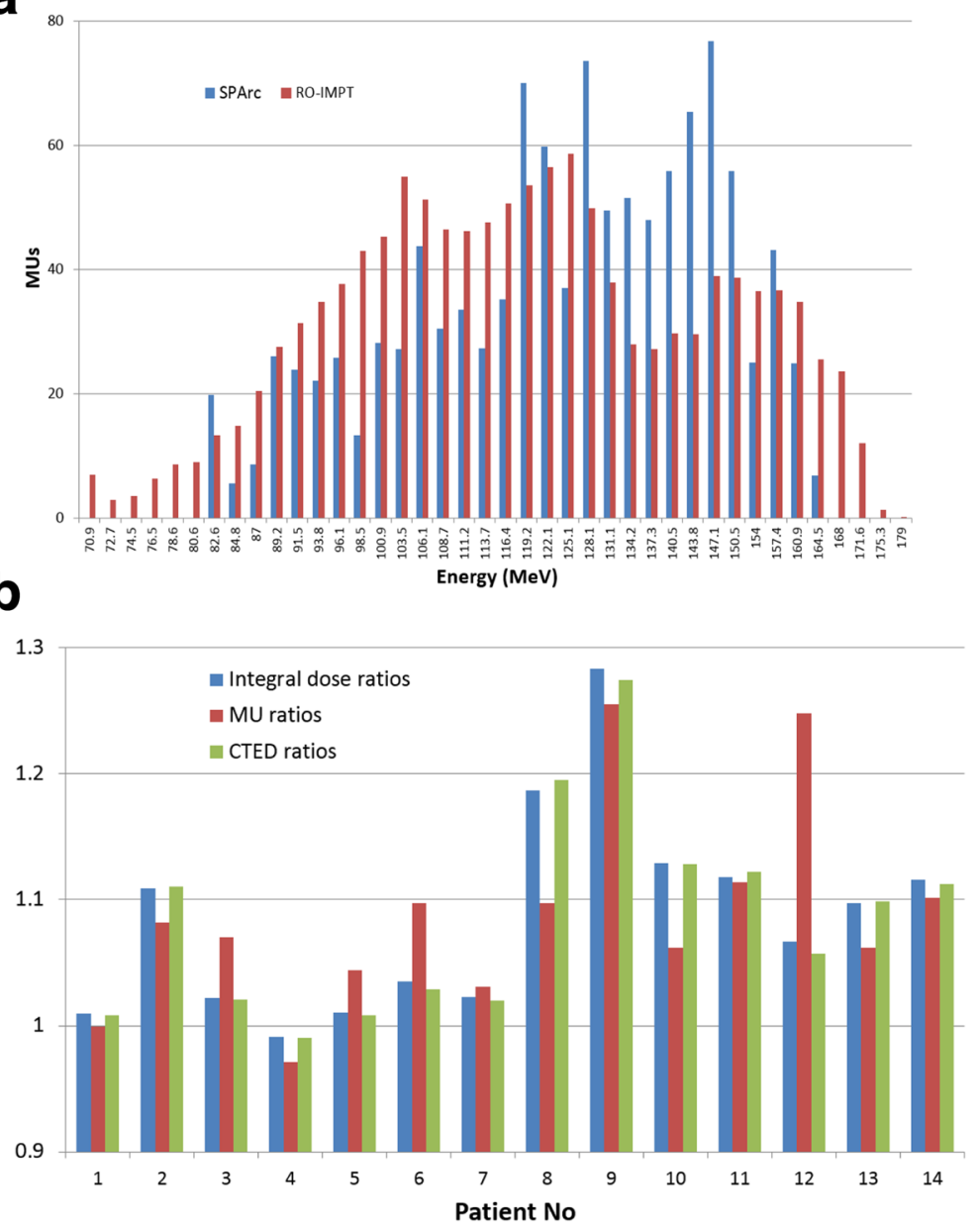

Fig. 5 a The distribution of MUs and energies for all the beamlets used between SPArc and RO-IMPT for patient 6. $\mathbf{b}$ The integral dose, MU, and the calculated total energy depositions (CTED) ratios for the fourteen patients between RO-IMPT and SPArc plans

depositions (CTED) were estimated as follows to evaluate the ratios between RO-IMPT and SPArc plans:

$$
C T E D=\sum_{i} M U i * E i / S(E i)
$$

where $M U i, E i, S(E i)$ are the monitor units, energy, and the corresponding proton stopping power for the energy in air for $i$ th energy layer. Such CTED ratios roughly estimate the total energy depositions between these two modalities. Figure $5 \mathrm{~b}$ shows the ratios among integral dose, total MUs, and CTED. The integral dose ratios match very well with the CTED ratios, which ultimately explain the reduction of integral body dose for the SPArc plan.

One of the major challenges with scanning beam proton therapy in treatment of lung cancer patients is the interplay effect between the spot scanning delivering sequence and the patient specific respiratory motion. Such interplay effect could lead to severe dose distortion especially in cases of large tumor motion, and could possibly be diminished by rescanning $[23,25,43,44]$. In this study for a fair comparison, we only compared the single-fraction 4D dynamic dose calculation without taking rescanning into account. The results demonstrated the ability of SPArc to reduce the interplay effect as shown in Fig. 4. This improvement could be explained by the washout effect of SPArc's hundreds of beam angles. Further studies are needed, however, evaluating different tumor motions and ELST simulating different patients and machine parameters to evaluate the interplay effect and SPArc more comprehensively. In routine clinical practice of using IMPT for motion target, rescanning technique is normally used in order to mitigate such interplay effects.

Moreover, it is important to mention that another potential benefit of using SPArc over the RO-IMPT plan could be the relative biological effectiveness (RBE) optimization. The RBE of 1.1 has been used historically and conservatively to ensure target coverage without taking into account the variation of RBE along the beam path. Paganetti et al. [45] has systemically analyzed the published experimental 
data and supported that there is an increase of RBE to 1.7 towards the distal falloff of the spread-out Bragg peak. Unlike traditional passive scatter proton therapy, IMPT has the potential to intentionally place the higher RBE around the Bragg peak inside the target volume to avoid any extra biological damage to the surrounding normal tissue. Furthermore, in case of SPArc, using hundreds of beam angles along with great energy layer selection magnetism would provide an even greater flexibility to locate the high RBE points inside the target volume. As indicated in Fig. 5a, even without biological optimization, the SPArc plan tends to avoid using very low and high energy beams, and therefore allows a greater probability of placing Bragg Peak into the target volume.

SPArc, as demonstrated here, shows significant potential to further improve the dosimetric outcome and therapeutic ratio in treating locally advanced NSCLC compared to RO-IMPT. Due to the limitations of current proton scanning beam systems, continuous gantry rotation with scanning delivery is still not available. Issues that need to be addressed in order to efficiently and safely implement SPArc for routine clinical use are: (1) developing a new rotational gantry which is capable of rotating around the iso-center while delivering pencil beam therapy with submillimeter accuracy; (2) designing new beamline control system to adjust and monitor the beam energy spectrum, spot position and spot profile during the gantry rotation, and (3) implementing a comprehensive SPArc quality assurance program.

\section{Conclusions}

SPArc is a robust and delivery-efficient proton spotscanning arc therapy technique which could potentially be implemented into routine clinical practice to further improve treatment outcomes in patients with locally advanced NSCLC.

\section{Abbreviations \\ AUC: Area Under the RVH curve; Cl: Conformality Index; CTED: Calculated total energy depositions; CTV: Clinical Target Volume; $\mathrm{D}_{0.03 c c}$ : Dose received by $0.03 c c$ volume; $D_{1 \%}$ : Dose received by $1 \%$ of volume; $D_{95 \%}$ : Dose received by $95 \%$ of volume; D99\%: Dose received by $99 \%$ of volume; DVHs: Dose Volume Histograms; ELST: Energy Layer Switching Time; GTV: Gross Target Volume; ID: Integral Dose; IMPT: Intensity Modulated Proton Therapy; IMRT: Intensity Modulated Radiation Therapy; ITV: Internal Target Volume; MU: Monitor Unit; NSCLC: non-small-cell lung cancer; OARs: Organ-at-risks; PSPT: Passive Scatter Proton Therapy; RBE: Relative Biological Effectiveness; RMSD: Root-mean-square dose; RO-IMPT: Robust Optimized Intensity Modulated Proton Therapy; RVHs: RMSD volume histograms; SPArc: Spot-Scanning Proton Arc; V5: Volume received at least 5Gy}

\section{Acknowledgements}

We would like to thank Guillaume Janssens and Antoine Pouppez from IBA (Ion Beam Application S.A. Louvain-la-Neuve, Belgium) for the discussions and supports in the IBA Proteus proton therapy system. This study is supported by research funding from IBA and Beaumont Health Herb and Betty Fisher Research Seed Grant Award.

\section{Funding}

The study is supported by research funding from lon Beam Application S.A. (Louvain-la-Neuve, Belgium) and Beaumont Health Herb and Betty Fisher Research Seed Grant Award.

\section{Availability of data and materials}

All data generated or analyzed during this study are included in this published article. Additional information is available from the corresponding author on reasonable request.

\section{Authors' contributions}

$\mathrm{XL}$ contribute to the acquisition, analysis and interpretation of data and draft and design the paper. PK contribute to revising the paper and providing clinical inputs; DY, AQ and JZ provide imaging acquisition and support; YH contribute to the statistical analysis; TG, IG and CS provided clinical inputs. $X D$, contribute to the design of the study, revise the draft and lead the research direction. All authors read and approved the final manuscript.

\section{Ethics approval and consent to participate}

The patient data used in this study is approved by Beaumont institutional IRB.

\section{Consent for publication}

Not applicable.

\section{Competing interests}

Xuanfeng Ding, Xiaoqiang Li and Di Yan has a pending patent based on the Spot-scanning proton arc (SPArc) algorithm.

\section{Publisher's Note}

Springer Nature remains neutral with regard to jurisdictional claims in published maps and institutional affiliations.

Received: 11 October 2017 Accepted: 20 February 2018

Published online: 27 February 2018

\section{References}

1. Siegel RL, Miller KD, Jemal A. Cancer statistics, 2016. CA Cancer J Clin. 2016;66(1):7-30

2. Furuse $\mathrm{K}$, et al. Phase III study of concurrent versus sequential thoracic radiotherapy in combination with mitomycin, vindesine, and cisplatin in unresectable stage III non-small-cell lung cancer. J Clin Oncol. 1999; 17(9):2692-9.

3. Zatloukal $P$, et al. Concurrent versus sequential chemoradiotherapy with cisplatin and vinorelbine in locally advanced non-small cell lung cancer: a randomized study. Lung Cancer. 2004;46(1):87-98.

4. Auperin A, et al. Meta-analysis of concomitant versus sequential radiochemotherapy in locally advanced non-small-cell lung cancer. J Clin Oncol. 2010;28(13):2181-90.

5. Fournel $\mathrm{P}$, et al. Randomized phase III trial of sequential chemoradiotherapy compared with concurrent chemoradiotherapy in locally advanced nonsmall-cell lung cancer: Groupe Lyon-saint-Etienne d'Oncologie ThoraciqueGroupe Francais de Pneumo-Cancerologie NPC 95-01 study. J Clin Oncol. 2005;23(25):5910-7.

6. Curran WJ Jr, et al. Sequential vs. concurrent chemoradiation for stage III non-small cell lung cancer: randomized phase III trial RTOG 9410. J Natl Cancer Inst. 2011;103(19):1452-60.

7. Grills IS, et al. Potential for reduced toxicity and dose escalation in the treatment of inoperable non-small-cell lung cancer: a comparison of intensity-modulated radiation therapy (IMRT), 3D conformal radiation, and elective nodal irradiation. Int J Radiat Oncol Biol Phys. 2003;57(3):875-90.

8. Kong FM, et al. High-dose radiation improved local tumor control and overall survival in patients with inoperable/unresectable non-small-cell lung cancer: long-term results of a radiation dose escalation study. Int J Radiat Oncol Biol Phys. 2005;63(2):324-33.

9. Schild SE, et al. Results of a phase I trial of concurrent chemotherapy and escalating doses of radiation for unresectable non-small-cell lung cancer. Int J Radiat Oncol Biol Phys. 2006;65(4):1106-11.

10. Yom SS, et al. Initial evaluation of treatment-related pneumonitis in advanced-stage non-small-cell lung cancer patients treated with concurrent chemotherapy and intensity-modulated radiotherapy. Int J Radiat Oncol Biol Phys. 2007;68(1):94-102. 
11. Bradley JD, et al. Primary analysis of the phase $\|$ component of a phase $\mathbf{I} / \|$ dose intensification study using three-dimensional conformal radiation therapy and concurrent chemotherapy for patients with inoperable nonsmall-cell lung cancer: RTOG 0117. J Clin Oncol. 2010;28(14):2475-80.

12. Liao ZX, et al. Influence of technologic advances on outcomes in patients with unresectable, locally advanced non-small-cell lung cancer receiving concomitant chemoradiotherapy. Int J Radiat Oncol Biol Phys. 2010;76(3):775-81.

13. Chang JY, et al. Significant reduction of normal tissue dose by proton radiotherapy compared with three-dimensional conformal or intensitymodulated radiation therapy in stage I or stage III non-small-cell lung cancer. Int J Radiat Oncol Biol Phys. 2006;65(4):1087-96.

14. Georg D, et al. Can protons improve SBRT for lung lesions? Dosimetric considerations. Radiother Oncol. 2008;88(3):368-75.

15. Zhang $X D$, et al. Intensity-modulated proton therapy reduces the dose to normal tissue compared with intensity-modulated radiation therapy or passive scattering proton therapy and enables individualized radical radiotherapy for extensive stage liib non-small-cell lung cancer: a virtual clinical study. I Int J Radiat Oncol Biol Phys. 2010;77(2):357-66.

16. Ding $X$, et al. A comprehensive dosimetric study of pancreatic cancer treatment using three-dimensional conformal radiation therapy (3DCRT), intensity-modulated radiation therapy (IMRT), volumetric-modulated radiation therapy (VMAT), and passive-scattering and modulated-scanning proton therapy (PT). Med Dosim. 2014;39(2):139-45.

17. Hoppe BS, et al. A phase 2 trial of concurrent chemotherapy and proton therapy for stage III non-small cell lung cancer: results and reflections following early closure of a single-institution study. Int J Radiat Oncol Biol Phys. 2016;95(1):517-22.

18. Lomax AJ, et al. The clinical potential of intensity modulated proton therapy. Z Med Phys. 2004;14(3):147-52

19. Lomax AJ. Intensity modulated proton therapy and its sensitivity to treatment uncertainties 1: the potential effects of calculational uncertainties. Phys Med Biol. 2008;53(4):1027-42.

20. Lomax AJ. Intensity modulated proton therapy and its sensitivity to treatment uncertainties 2: the potential effects of inter-fraction and interfield motions. Phys Med Biol. 2008;53(4):1043-56.

21. Dowdell $\mathrm{S}$, et al. Interplay effects in proton scanning for lung: a 4D Monte Carlo study assessing the impact of tumor and beam delivery parameters. Phys Med Biol. 2013;58(12):4137-56.

22. Grassberger $\mathrm{C}$, et al. Motion interplay as a function of patient parameters and spot size in spot scanning proton therapy for lung cancer. Int J Radiat Oncol Biol Phys. 2013;86(2):380-6.

23. Kardar $L$, et al. Evaluation and mitigation of the interplay effects of intensity modulated proton therapy for lung cancer in a clinical setting. Pract Radiat Oncol. 2014;4(6):e259-68.

24. Knopf AC, Hong TS, Lomax A. Scanned proton radiotherapy for mobile targets-the effectiveness of re-scanning in the context of different treatment planning approaches and for different motion characteristics. Phys Med Biol. 2011;56(22):7257-71.

25. Li Y, et al. On the interplay effects with proton scanning beams in stage III lung cancer. Med Phys. 2014;41(2):021721.

26. Pflugfelder D, Wilkens JJ, Oelfke U. Worst case optimization: a method to account for uncertainties in the optimization of intensity modulated proton therapy. Phys Med Biol. 2008;53(6):1689-700.

27. Unkelbach J, et al. Reducing the sensitivity of IMPT treatment plans to setup errors and range uncertainties via probabilistic treatment planning. Med Phys. 2009;36(1):149-63.

28. Fredriksson A, Forsgren A, Hardemark B. Minimax optimization for handling range and setup uncertainties in proton therapy. Med Phys. 2011;38(3):1672-84.

29. Liu W, et al. Robust optimization of intensity modulated proton therapy. Med Phys. 2012;39(2):1079-91.

30. Chen $\mathbf{W}$, et al. Including robustness in multi-criteria optimization for intensity-modulated proton therapy. Phys Med Biol. 2012;57(3):591-608.

31. Liu W, et al. Influence of robust optimization in intensity-modulated proton therapy with different dose delivery techniques. Med Phys. 2012;39(6):3089.

32. Liu W, et al. Impact of respiratory motion on worst-case scenario optimized intensity modulated proton therapy for lung cancers. Pract Radiat Oncol. 2015;5(2):e77-86.

33. Ding $X$, et al. Spot-scanning proton arc (SPArc) therapy: the first robust and delivery-efficient spot-scanning proton arc therapy. Int J Radiat Oncol Biol Phys. 2016;96(5):1107-16.
34. Paganetti $\mathrm{H}, \mathrm{Yu} \mathrm{CX}$. Photon radiotherapy has reached its limit in terms of catching up dosimetrically with proton therapy. Med Phys. 2016;43(8):4470.

35. Ding $X$, et al. Have we reached proton beam therapy dosimetric limitations? - A novel robust, delivery-efficient and continuous spot-scanning proton arc (SPArc) therapy is to improve the dosimetric outcome in treating prostate cancer. Acta Oncol. 2017;57(3):435-37.

36. Murshed $\mathrm{H}$, et al. Dose and volume reduction for normal lung using intensity-modulated radiotherapy for advanced-stage non-small-cell lung cancer. Int J Radiat Oncol Biol Phys. 2004;58(4):1258-67.

37. Quan EM, et al. A comprehensive comparison of IMRT and VMAT plan quality for prostate cancer treatment. Int J Radiat Oncol Biol Phys. 2012:83(4):1169-78.

38. Liu W, et al. Effectiveness of robust optimization in intensity-modulated proton therapy planning for head and neck cancers. Med Phys. 2013; 40(5):051711.

39. Kang $M$, et al. A study of the beam-specific interplay effect in proton pencil beam scanning delivery in lung cancer. Acta Oncol. 2017;56(4):531-40.

40. Bradley JD, et al. Standard-dose versus high-dose conformal radiotherapy with concurrent and consolidation carboplatin plus paclitaxel with or without cetuximab for patients with stage IIIA or IIIB non-small-cell lung cancer (RTOG 0617): a randomised, two-by-two factorial phase 3 study. Lancet Oncol. 2015;16(2):187-99.

41. Ramroth J, et al. Dose and Fractionation in Radiation Therapy of Curative Intent for Non-Small Cell Lung Cancer: Meta-Analysis of Randomized Trials. Int J Radiat Oncol Biol Phys. 2016:96(4):736-47.

42. Chun SG, et al. Impact of intensity-modulated radiation therapy technique for locally advanced non-small-cell lung cancer: a secondary analysis of the NRG oncology RTOG 0617 randomized clinical trial. J Clin Oncol. 2016;0(0):JCO691378.

43. Kraus KM, Heath E, Oelfke U. Dosimetric consequences of tumour motion due to respiration for a scanned proton beam. Phys Med Biol. 2011;56(20):6563-81.

44. Lambert J, et al. Intrafractional motion during proton beam scanning. Phys Med Biol. 2005;50(20):4853-62

45. Paganetti H. Relative biological effectiveness (RBE) values for proton beam therapy. Variations as a function of biological endpoint, dose, and linear energy transfer. Phys Med Biol. 2014;59(22):R419-72.

\section{Submit your next manuscript to BioMed Central and we will help you at every step:}

- We accept pre-submission inquiries

- Our selector tool helps you to find the most relevant journal

- We provide round the clock customer support

- Convenient online submission

- Thorough peer review

- Inclusion in PubMed and all major indexing services

- Maximum visibility for your research

Submit your manuscript at www.biomedcentral.com/submit
C) Biomed Central 